\title{
PERIODISMO EN LÍNEA \\ Y DESARROLLO DE BLOGS COMO \\ ALTERNATIVA DE EXPRESIÓN \\ INFORMATIVA*
}

\author{
María de la Luz Casas Pérez ${ }^{* *}$
}

Recibido: 9 de abril de 2010. Aceptado: 23 de mayo de 2010.

\section{RESUMEN}

El término "blog" proviene de las palabras Web y Log, y se ha convertido en una herramienta de recuento de historias paralelas, ya que a través de ellos es posible visualizar distintas interpretaciones a un mismo acontecimiento.

Si bien el origen de los blogs es personal, a últimas fechas los medios periodísticos en línea han colocado blogs como recurso de interacción entre sus lectores. Esto les ha arrojado retroalimentación, y ha permitido obtener una sensibilidad directa de la opinión pública en formación y promueve nuevos temas a insertarse en la agenda.

El presente trabajo revisa los blogs como alternativas de información al recurso periodístico, analizando el comportamiento de los blogs insertados en diversos periódicos y sitios de información periodística en línea.

Palabras clave: 6 logs, periodismo digital.

Este artículo de investigación científica y tecnológica se desarrolla en las líneas de comunicación política y medios de comunicación que desarrolla la autora.

Licenciada en comunicación. Magíster en comunicación. Doctora en Ciencias Políticas. Tecnológico de Monterrey. Campus Cuernavaca. México.E-mail: marilu.casas@itesm.mx 


\title{
ONLINE JOURNALISM AND DEVELOPMENT OF BLOGS AS AN ALTERNATIVE OF INFORMATION TECHNOLOGY EXPRESSION
}

\begin{abstract}
The term "blog" comes from the words Web and Log and it has recently become a tool to retell parallel stories, since they allow their audience to visualize different interpretations of the same event.

Although blogs were personal in origin, lately online media journalists are using blogs as an additional information source, as well as an interaction resource for readers. For media, blogs give useful feedback for the journalistic agenda also promoting new subjects to be discussed.

This article offers a review on blogs as an alternative source of information for journalistic media, while analyzing the behavior of blogs from well-known journals and web sites. Its results show some of the most claimed contents communicated by these media.
\end{abstract}

Key words: blogs, online journalism. 


\section{INTRODUCCIÓN}

El origen de los blogs data de los años 90 y algunos lo confieren a Jorn Barger, editor de Robot Wisdom y conocedor de Internet, el haber acuñado el término Weblog (Wikipedia, 2009). Según Blood (2000), los blogs aparecieron por primera vez como una novedad en la utilización de aplicaciones interactivas a través de la computadora, con la página What's New Page del National Center for Supercomputing Applications de la Universidad de Illinois en 1993 (Blood en Walker, 2005). Posteriormente se comienza a hablar de los blogs como recursos de información, sin embargo, no se empieza a hablar de la generalización de los blogs sino hasta 1997-1998 cuando se proporciona un listado de páginas accesibles en las que se discuten diversos temas y que fue recopilada fundamentalmente gracias a los esfuerzos de David Winer y Peter Merholz.

El término Weblog lo acuña Jorn Barger precisamente en 1997 para referirse a los ingresos o visitas que determinados usuarios hacían a páginas web, quienes dejaban un comentario o un registro de visita. Para 1999, Merholz sugería la expresión we-log, indicando la práctica de entrar y dejar este tipo de registros. (Branum, 2001).

Los blogs se generalizarían a partir de 1999 con la inauguración oficial de portales dedicados a la publicación de blogs, como Eaton Web Portal, y con el lanzamiento comercial de Blogger, el servicio gratuito de publicación de weblogs.

En sus inicios, los blogs fueron utilizados como una herramienta de uso personal en el que los visitantes plasmaban sus ideas y comentarios acerca de algún tema en particular, proporcionando acceso abierto o restringido a una comunidad específica de lectores. No obstante, el blog ha evolucionado hasta convertirse en un recurso informativo de enorme valor para distintos propósitos. De manera institucional, por ejemplo, las empresas han recurrido a los blogs en sustitución de las revistas internas, a fin de establecer una conexión más cercana con sus em- pleados y clientes, mientras que por otro lado, reconociendo el enorme valor de los blogs como ventanas hacia la opinión pública, los medios de comunicación tradicionales, especialmente los noticiosos, han establecido blogs con el propósito específico de abrir las noticias a la interacción e intercambio de comentarios entre los lectores, radioescuchas o telespectadores. Desde esta perspectiva, el blog se ha convertido incluso en una importante arma, a través de la cual se siembran los asuntos a discutir en la agenda política (Drezner, 2004).

\section{JUSTIFICACIÓN DEL ESTUDIO}

En todas las épocas quién escribe qué, sobre qué o sobre quién, ha sido siempre un asunto de interés para las élites o los círculos de la intelectualidad social o política.

La opinión privada traspasó los límites de la intimidad para insertarse en la arena política, mientras las sociedades cortesanas divulgaron sus opiniones de viva voz llevando la información de un lado a otro en tertulias o cafés. Cuando las cartas y diarios privados de la aristocracia invadieron las calles llevando el chisme al pueblo, la vida de los importantes se convirtió en temática del corrillo popular, e incluso llegó a plasmarse en diarios y volantes de difusión restringida. La aparición de los medios de comunicación, primariamente censurados por el poder político, inhibió esta práctica que venía desarrollándose desde la Edad Media.

Hoy el blog viene a recuperar electrónicamente la tradición del diario, de la bitácora de viaje, del álbum de recuerdos y de tantos otros recursos de recuperación de información, convirtiéndose en un recurso de gestión y de identificación con el grupo de referencia.

En los blogs podemos encontrar información de todo tipo, reflejando de alguna manera la forma de sentir, el diálogo y la propuesta de soluciones ante problemáticas sensibles que aquejan a la sociedad contemporánea. 
Los blogs son uno de los recursos más socorridos por los internautas hoy en día y junto con otros espacios de relación, se consideran uno de los más favorecidos por los interesados en participar en lo que hoy en día se considera la "red social" virtual.

La prensa escrita hoy se sirve de los blogs, ya que ha comprobado que pueden ser un recurso mucho más eficiente que ella misma para convocar a la opinión pública y tomar temas para trasladarlos a las principales planas de los periódicos. De hecho, como algunos investigadores sostienen, el Presidente de los Estados Unidos, Barack Obama, es el primer producto de la discusión de fenómenos de interés público a través de las redes sociales, como bien analiza el Massachussets Institute of Technology, en su Technology Review (Talbott, 2008).

¿Qué tan autónomos son los blogs? ¿En qué medida responden a los requerimientos de participación de una sociedad civil que clama por espacios de interacción y promoción de la opinión pública? ¿Se trata, por el contrario, de espacios conducidos políticamente, orquestados para alentar la gestión de movimientos de presión? ¿Por qué puede, en un momento dado, un medio de comunicación tradicional, como un periódico en línea o un noticiero de televisión, decidir abrir un blog para interactuar directamente con su público? ¿Qué tan abundante es la información de estos blogs o qué tanto son consultados y con referencia a qué tipo de temáticas?

Es por ello que en este estudio hemos decidido abocarnos al análisis del blog, no como una manifestación de expresión individual, sino como espacios de relación deliberadamente orquestados por instituciones de medios para orientar y promover expresiones que posteriormente serán utilizadas como motor de guía para procesos de opinión pública.

\section{METODOLOGÍA}

A fin de contribuir a la organización y mejor entendimiento del funcionamiento de los blogs y de la manera en que la prensa impresa ha hecho uso de este material, se recurrió a la clasificación que proporciona Fumero (2005) basado en Wacka (2004) y Branum (2001).

La metodología establece que puede haber diferentes tipos de principios bajo los cuales funciona un blog y pueden responder a criterios de orden social entre los cuales están: una motivación personal o profesional; o bien utilizarlos con fines de promover a una organización o corporación, que pueden tener objetivos de naturaleza externa (ventas, relación, imagen de marca), o bien internos (gestión del conocimiento, colaboración, cultura corporativa).

Por otra parte, pueden tener varios propósitos: horizontales (de propósito general), o verticales (con un propósito más específico). Los blogs también varían de acuerdo con el ámbito de su aplicación, ya que hay los que aceptan temas políticos, organizacionales o personales, deportivos, culturales, entretenimiento, religión, farándula, etc. De acuerdo con su carácter informacional, podemos incluso encontrar blogs de texto, audioblogs, fotoblogs, o videoblogs, dependiendo del tipo de información que recogen.

Los blogs de los medios periodísticos son en su mayoría textuales; no obstante, hay medios como los noticieros televisivos que hacen gala y aprovechamiento de la posibilidad que tienen para acumular información en vídeo, y dejar huella y registro de otro tipo de información. Los canales de televisión que cuentan con blogs también hacen uso de audio y fotos. Los temas que utilizan los medios informativos periodísticos tienen que ver no solamente con la noticia del día, sino también con notas secundarias sobre tecnología, cultura, gente, música, literatura, deportes, política, negocios, economía, sociedad, arte y otros temas.

El presente trabajo se desarrolló a partir de una metodología mixta que permitió la recuperación de la caracterización de lo que es un blog, su clasificación a partir de diversos criterios de 
orden social, estructural, e informacional, su cuantificación, la componente tecnológica, sindicación de contenidos y sus plataformas de publicación, entre otros temas.

Los criterios utilizados para la clasificación fueron los siguientes:

1. Criterios de orden social:

1.1. Motivación: Ofrecen espacios para crear blogs personalizados con temas libres

1.2. Profesional: Ofrecen publicaciones especializadas y servicios, aprovechando las características de los blogs como herramienta de colaboración y comunicación

1.3. Organizativa/corporativa:

1.3.1. Externos, cuyo propósito se vincula con la organización para promover:

1.3.1.1. Ventas

1.3.1.2. Relación

1.3.1.3. Imagen de marca

1.3.2. Internos, cuyo propósito es buscar alguno de los siguientes objetivos:

1.3.2.1. Gestión del conocimiento

1.3.2.2. Colaboración

1.3.2.3. Cultura corporativa

1.4. Propósito, entendido como el sentido de la divulgación de información en el blog, cuyos contenidos son:

1.4.1. Horizontales. Blogs sobre temas libres que son intercambiados por personas que tienen las mismas características.

1.4.2. Verticales. Blogs sobre temas específicos cuyo contenido es intercambiado con distintos niveles de profundidad.

1.5. Ámbito de aplicación: relacionado con las temáticas a las cuales impactan los intercambios dentro del blog, que pueden ser de naturaleza varia: política, organizacional, profesional y personal, o referirse a cuestiones de interés en el campo de los deportes, cultura, entretenimiento, política, religión, medio artístico, y más.
1.6. Autoría: referente al autor o productor del blog. Los blogs pueden ser de autoría:

1.6.1. Individual

1.6.2. Colectiva

2. Criterios de orden estructural. Los blogs no solo son recuentos de las expresiones de algunas personas, sino que también hacen alusión a información publicada por otros. La mayoría de los blogs disponen de naturaleza hipertextual y carácter conversacional.

2.1. Naturaleza hipertextual. Dentro de los blogs que nos remiten a otros blogs, encontramos los siguientes:

2.1.1. Intrablog. Un blog al que se hace referencia dentro de otro blog y que se encuentra en el mismo espacio de discusión.

2.1.2. Extrablog: Un blog fuera del blog.

2.1.3. Isoblog: Se refiere a blogs elaborados por grupos específicos con estándares de información ISO en cuanto a seguridad.

2.2. Carácter conversacional: Son blogs que reportan el intercambio de expresiones entre los lectores, que se convierten a sí mismo en co-autores del blog.

3. Criterios de orden informacional

3.1. Contenidos

3.1.1. Blogs textuales/estándar: solo presentan texto

3.1.2. Audioblogs: contienen audio

3.1.3. Fotoblogs: proporcionan fotografías

3.1.4. Moblogs: son blogs que permiten ser consultados desde dispositivos móviles.

3.1.5. Videoblogs: incluyen vídeo.

El trabajo de campo se hizo a partir de indagación en la red sobre la base de tres periódicos mexicanos reconocidos (Excélsior, El Universal y el Reforma) y de tres cadenas de televisión mexicanas igualmente reconocidas (Once TV, TV Azteca y Televisa). No hubo un criterio definido para la selección de medios, más allá de tomar en consideración que todos ellos hicieran uso regular del recurso de los blogs. 
La información nos permitió generar una base de datos constituida por una matriz en la que se registró la información obtenida durante los meses de enero a junio del 2009, en la cual se cruzó información relativa a la naturaleza y criterios de los blogs encontrados, contra el carácter institucional o de debate de cada uno de los blogs encontrados en los medios a los que se hizo referencia previamente.

\section{RESULTADOS}

Una de las principales problemáticas para analizar el tema radica en que el objeto de estudio cambiaconstantementeynoesposibledeterminar con precisión sus características. En este caso, el período en el que se estuvieron observando los blogs de los sitios indicados anteriormente fue de poco más de seis meses, con el propósito de corroborar si era factible registrar un patrón constante en el sitio observado. De esta manera, se hizo una evaluación de los sitios, tomando en consideración la naturaleza del medio, de forma que inicialmente se realizó una observación de blogs, agrupándolos por el tipo de sitio en el que se publicaron. Para el registro se elaboró una matriz por tipo de medio de comunicación, que permitió realizar un inventario del tipo de elementos que cada blog ofrece de acuerdo con la tipología mencionada anteriormente. Este registro contenía una descripción simple de las características de cada blog y del tipo de interacción que permite con los usuarios.

Los resultados de los blogs analizados que correspondieron a las estaciones de televisión demostraron que en su mayoría estos blogs se caracterizan por abordar los temas de una manera superficial. Las temáticas abordadas son deportes, farándula, salud, entretenimiento, astrología o bienestar personal.

En cambio, al hacer una evaluación de los blogs publicados en sitios de periódicos en línea, se encontró que los blogs reconocen los intereses de los autores, o los de las instituciones en las que laboran. En general, el periódico contrata a una personalidad reconocida para que escriba sobre temas de interés y analice los eventos de actualidad.

Es importante señalar que algunos de los blogs que eventualmente reciben posada en un medio tradicional han sido creados a iniciativa de un navegante asiduo al sitio de Internet, que desea dar a conocer sus puntos de vista y ofrece la participación periódica a través de un blog.

En algunos casos los blogs pueden ser intercambio publicitario con una institución, o bien ser pagados a un externo. Esta información se obtiene observando el registro y firma de quienes participan como autores y la declaración que hacen acerca de los propósitos de su blog. Por ejemplo, podemos encontrar blogs relativos a finanzas, desarrollados por una asociación de contadores o consultores en finanzas, un blog relativo a cuestiones de protección al consumidor, otro de nutrición, de educación, etc.

Los blogs se distinguen por su temática y generalmente aparecen relacionados con la programación del canal de televisión, o bien con las secciones tradicionales del periódico impreso.

La cantidad de autores que abordan temas específicos también indica la importancia que el periódico y sus lectores le otorgan a determinadas temáticas. Destaca la influencia del tipo de medio y de la naturaleza género periodístico y la forma en la que éstos impactan a las temáticas a las que se les brinda un espacio a través de los blogs. Así, si una temática está vigente o es muy importante para el establecimiento de la agenda periodística es muy probable que otorgue espacio a uno o varios blogs que proporcionen información adicional sobre el tema. Sin embargo, también puede suceder que la televisora o el periódico introduzcan temas nuevos en la agenda y los abran para la discusión. Esta última situación se presenta con mucho más frecuencia que en los sitios de periódicos.

En nuestro análisis por tipo de medio, se observó que las televisoras tienden a utilizar más su 
espacio de Internet ofreciendo pequeñas cápsulas o fragmentos de vídeo sobre su programación o sobre aspectos importantes de sus noticieros y no los abren tanto para la discusión libre a través de blogs. En el caso de blogs existentes en empresas de televisión, éstos son utilizados principalmente para que los espectadores interactúen con alguna personalidad, actor o cantante, o bien para que envíen preguntas, sugerencias y comentarios específicos sobre la programación. Lo que es más, Televisa explota crecidamente la herramienta del blog, que Televisión Azteca o Canal Once.

No todos los sitios pertenecientes a televisoras tienen uso de blogs, ni los aprovechan de la misma manera. Ocasionalmente alguna televisora abre un blog, regularmente relativo a algún programa de deportes o de farándula, para dar oportunidad de que los televidentes expresen sus opiniones sobre el programa. El resto de los sitios interactivos que aparecen disponibles solamente permiten enviar correos o comentarios sobre la programación en turno.

Los periódicos en línea que fueron analizados en este estudio mostraron un especial interés por aprovechar la herramienta de los blogs para ir buscando lectores especializados, e ir construyendo más sitios que les permitan desarrollar un vínculo específico con nuevos nichos de mercado.

De tal suerte es posible encontrar blogs con temáticas específicas para jóvenes, concretamente jóvenes universitarios, pero también es posible encontrar blogs para amantes de la música o del arte.

En el caso de los autores de blog, no hay un perfil definido. Normalmente se trata de personalidades que se han dado a conocer a través del mismo medio, o bien a través de otros medios. También es posible encontrar tanto a académicos o investigadores, como a artistas, personalidades de la política o miembros de asociaciones reconocidas.
Una característica del blog es que puede variar con relación al estilo de redacción y al nivel de especialización. Los hay redactados en un estilo sumamente coloquial, y otros referidos a cuestiones más especializadas.

De manera particular, los blogs que aparecen publicados en periódicos mexicanos en línea hacen un mejor aprovechamiento de la palabra escrita. Destacan, además, el número de colaboradores y blogs de distintas temáticas que son publicados por los periódicos nacionales que aparecen en la muestra. También enfatiza la posibilidad de la generación de espacios de discusión de élite, a los que solamente tienen acceso aquellos que son conocedores de una determinada tendencia o estilo, o bien quienes gozan de un registro especial como suscriptor del periódico en línea. Lo anterior indica que, de la misma manera que el periódico impreso, segmenta a sus lectores dependiendo del tipo de sección a la que hace referencia, los lectores de blogs siguen de manera fiel a los autores que normalmente atienden los temas de su interés.

Destaca el hecho de que la temática de los blogs no solamente se refiere a cuestiones de tecnología de punta, sino que también ofrece la oportunidad de expresar lo que se piensa o lo que se siente sobre determinado tema.

En los periódicos en línea analizados fue especialmente interesante observar la opinión de los lectores respecto de temas relativos a economía y a política. La economía, la política y los deportes son las áreas más desarrolladas en el ámbito de los blogs. Los temas más socorridos en el período en cuestión fueron indudablemente: la crisis económica, la epidemia de influenza (acontecida en México en este período) y las elecciones.

Es importante hacer mención que si bien algunos de los blogs provienen de la pluma de analistas y escritores reconocidos, lo que indudablemente proporciona un prestigio adicional al 
periódico, también se encontraron espacios de expresión libre. Estos son importantes, ya que en ellos se brinda a los lectores la oportunidad de decir lo que sienten o piensan de manera libre. El número de respuestas a cada entrada proporciona además una dimensión del valor y popularidad que un blog provoca. En ocasiones los periódicos publican todos los comentarios que generan los blogs, y en otras simplemente publican los más recientes.

Por otra parte, cuando el blog es de autoría personal de un experto, éste muy probablemente será quien decida qué es lo que deja ver al resto de sus lectores y qué no. En ese sentido, el autor del blog genera una especie de censura o autocensura.

\section{ANÁLISIS}

Orihuela (2006) utiliza la siguiente clasificación para referirse a las personas que visitan blogs: a) parroquianos, b) turistas y c) surfers; esto es dependiendo del tipo de uso que cada visitante hace a un blog.

Los parroquianos se consideran parte de una comunidad; los turistas son visitantes fugaces, mientras que los surfers simplemente son navegantes de paso que simplemente andan viendo lo que se publica.

Esta clasificación ahonda en lo que algunos analistas destacan como la diferencia fundamental entre la interacción y la interactividad, entendiendo por interacción la capacidad que nos da un blog al simplemente hacer un clic en un espacio o una liga para encontrar información oportuna, trascendente o novedosa sobre un tema particular, o bien ser sitios verdaderamente interactivos que permiten a quienes los visitan no únicamente la lectura, sino también la participación. Una característica importante de los blogs que fueron analizados como parte de esta muestra es su diferencia en cuanto a las características de interacción o interactividad que promueven.
Las cadenas mexicanas de televisión, a excepción de Televisa, utilizan sus blogs simplemente para informar acerca de su programación, ofrecer la oportunidad de subir un comentario; en cambio, los blogs que aparecen en los sitios de la prensa mexicana en línea ofrecen verdaderos espacios de discusión entre sus usuarios. Podríamos decir entonces, que mientras las televisoras ofrecen cierta posibilidad de interacción, la prensa en línea es la que verdaderamente se abre a la posibilidad a la interactividad.

Tanto las cadenas de televisión como los medios de prensa escrita recurren a blogs, la mayoría de ellos pertenecientes a la categoría de "relación", pues el motivo de extender los métodos tradicionales de comunicación entre estos medios y sus receptores fue el de crear un vínculo entre las empresas, sus televidentes o lectores.

Los blogs periodísticos son en su mayoría textuales, al igual que los blogs televisivos en línea. Sin embargo, hay algunos que cuentan con ligas a vídeos o audios relacionados con el tema puesto a discusión. Los canales de televisión que cuentan con blogs al aire hacen más uso del audio, vídeo y fotografías.

Los temas tratados en los blogs tanto de medios televisivos como periodísticos son básicamente: tecnología, cultura, gente, música, cine, literatura, deportes, política, negocios, economía, artistas, sociedad, nacional y temas libres.

Televisión Azteca y Canal 11 colocan blogs que se relacionan exclusivamente con la programación del canal.

Una observación puntual hecha por el equipo de investigación encontró que los blogs del periódico El Universal tratan temas diversos, desde economía y política, hasta salud y religión. Un dato interesante es que realmente se presenta una interacción entre los especialistas y sus lectores. Un ejemplo de ello es el blog Nutrición... ni bueno ni malo, en donde los temas que se tratan son respondidos y comentados abiertamente. 
Por su parte, el periódico Reforma contiene blogs que son exclusivos y para los cuales se requiere ser suscriptor del periódico.

La literatura académica al respecto anticipa que para explicar el uso y apropiación de los blogs es necesario desarrollar un nuevo cuerpo teórico que permita explicar los usos y gratificaciones de los participantes en blogs. (Branum, 2001). Se presupone, por ejemplo, que así como en su momento la Teoría de la Agenda Setting intentó explicar la forma en que los medios establecen las temáticas para la agenda de la discusión, de la misma manera los blogs orientan la discusión de manera diferenciada dependiendo de la naturaleza de cada uno. Por ejemplo: un blog desarrollado de manera libre establece la agenda para sus lectores dependiendo de las lecturas que hace, de las referencias o las fuentes a las que cita. No obstante la pregunta indicada es: ¿quién establece la agenda: el blogger? Es decir ¿el autor del blog? ¿O la fuente en la cual su blog aparece? (Klopfenstein, en Branum, 2001).

Por otra parte, si el blogger "recicla" ligas hacia otros sitios en la red, o a otros blogs, de alguna manera lo que hace es definir la agenda a partir de asignar prioridades de acuerdo con sus preferencias. En este sentido algunos autores sugieren que el fenómeno del blogging debería ser analizado a partir de una teoría no de flujo de comunicación en dos pasos (Two-step flow), sino de múltiples pasos (multi-step flow), a partir del cual la agenda se establece dependiendo del número de referencias o citas que un blog recibe dentro de otros blogs (Branum, 2001).

Dado que algunos de los principales periódicos en línea aprovechan la fama o el prestigio de quienes escriben en su blog (por ejemplo el prestigio de Jesús Silva-Herzog Márquez, o la fama de Soledad Loaeza), el fenómeno del blogging también podría ser analizado a partir de una Teoría del Líder de Opinión, pero evolucionada, en donde el liderazgo se manifiesta no a partir de lo que los medios dicen del líder, o de la interpretación que el líder da de las noticias y que transmite más adelante, sino de un liderazgo al que el lector accede a partir de leer las experiencias y opiniones del líder en su blog.

El autor de un blog normalmente es una persona que monitorea la agenda de los medios con mayor frecuencia que otros lectores o usuarios de los medios. Ello le permite comentar, profundizar o someter a debate ciertas temáticas y si su blog es favorecido por buen número de lectores o de consultas, es mucho más probable que él mismo se convierta en un líder de opinión. Utilizando la definición de lo que es el liderazgo de opinión según Robinson, (1976), Clark, (2001) indica que leer el sitio creado por un blogger famoso genera estatus para quien lo lee, y Lassica (2001) supone que es la comunidad, a través del número de visitas que recibe un blog, quien avala o certifica la cualidad de liderazgo del blogger (Robinson, Clark y Lassica en Branum, 2001). A su vez, Blood (2001) supone que los comentarios colocados en un blog por parte de los lectores, proporcionan al blogger una idea clara del apoyo con el que cuentan sus opiniones.

Werbach (2001) indica quelos autores de blogs de estilo libre también operan como sistemas alternativos a los guardabarreras (gatekeepers), ya que suplen de alguna manera los criterios de censura que operaban naturalmente en los medios impresos o audiovisuales tradicionales. No obstante, el propio blogger se convierte en un censor o guardabarreras, ya que él mismo requiere ser un lector asiduo de la información publicada previamente por los medios masivos, haber hecho una selección precisa de aquello que vale la pena promover, y fungir asimismo como guía de aquellos que no tienen la información de primera mano.

Dvorak (2002) por su parte indica que la necesidad de escribir blogs puede deberse a diferentes razones: búsqueda de gratificación, necesidad de eliminar la frustración, búsqueda de aprobación social y necesidad de dejar huella a través de la expresión individual. En todo caso, quienes publican blogs, o escriben y comentan en 
ellos, están proporcionando información al resto, que puede o no compartir sus opiniones, pero que igualmente puede acabar siendo influenciado por ellas.

La producción, mantenimiento y monitoreo de los blogs, a su vez, requiere de cierto entrenamiento. En última instancia, después de todo este proceso de selección, edición y comentario, cuando el blogger genera una nueva entrada, tiene que hacerlo de manera que el lector del blog encuentre propuestas mucho más interesantes que las que ofrecen los medios tradicionales. Los contenidos atractivos del blog pueden consistir en nuevas interpretaciones de la información tradicional que proporcionan los medios; ligas y vínculos a información adicional (fotografías, imágenes o videos), comentarios del blogger o de otros usuarios que coinciden directamente con las expectativas de quien los lee o la posibilidad real de someter la opinión personal de uno como contribución directa al blog.

Lo que sí es importante considerar es que los blogs que aparecen publicados en los medios tradicionales reciben el aval del medio del que son huéspedes, (ya sea un periódico o una estación de televisión en este caso); que quienes escriben en ellos probablemente sean reporteros o periodistas adscritos a ese mismo medio, que se ejercitan en otro tipo de género de difusión de contenidos, periodísticos y no periodísticos, y que contribuyen de alguna manera a formar la opinión de sus lectores.

Por lo que puede observarse, el fenómeno del blogging impone un carácter nuevo y diferente al producto periodístico en línea, generando para los lectores una oportunidad de participación directa de la que no disponían los medios tradicionales. Es así que el blog representa un puente entre el lector o la audiencia y los medios en los cuales éstos se asientan.

\section{CONCLUSIONES}

Los blogs se han convertido en un nuevo espacio de relación.
La construcción de sentido en los blogs se desarrolla a través de encadenamientos comunicativos de autoría individual o colectiva, que al vincularse unos a otros establecen tipos de construcción comunicativas interesantes y complejas como lo menciona Pérez Salazar (2009). El blog es, por tanto, un fenómeno de interés que debe analizarse. En su estructura no solamente incluye un conjunto de información activa, a través de la cual es factible ordenar, observar y derivar las estructuras de argumentación mediante las cuales se ordena y plantea un discurso, sino que también dirigen la opinión a través de sugerir navegación relacionada. En ese sentido es de suma importancia entender que se trata de herramientas de formación de opinión sumamente útiles.

La pregunta inicial de investigación que guió este trabajo sugería que los medios de comunicación tradicionales, particularmente la televisión y los periódicos en línea, utilizarían de alguna manera sus sitios en Internet aprovechando las bondades de la interacción, particularmente a través de blogs, a fin de vincularse de manera más directa con sus telespectadores y lectores. Los resultados sugieren que a diferencia de la prensa, la televisión no ha logrado o no ha podido aprovechar la herramienta de los blogs, quizá debido a la naturaleza más visual y dinámica de sus audiencias. En cambio, los periódicos en línea, en muchos casos, se encuentran ya en el proceso de construcción de espacios de relación y de discusión pública a través de la generación de híperacontecimientos que se presentan a sí mismos como nuevos detonadores de la discusión.

Resulta interesante, además, conocer las expectativas y necesidades de interacción que los usuarios de blogs manifiestan, a través del registro de entradas específicas a los anclajes y nodos particulares de un blog, ya sea a través de preguntas puntuales que el creador del blog lanza a sus lectores, o bien a través de enlaces o sugerencias de lecturas adicionales que conducen a ligas, vídeos, imágenes y fotografías que se proporcionan como información derivada. 
El análisis de algunos de los comentarios proporcionados por los usuarios de los blogs observados sugiere la necesidad que tienen los lectores de interactuar con las celebridades, los grandes autores y los creadores de nuevas tendencias. Esto parece indicar que las audiencias ya no desean ser pasivas, y que en términos generales agradecen la oportunidad de ser parte de la comunicación y dejar su propia huella en la Internet.

Otra cuestión que se presenta como relevante es la necesidad de encontrar nuevos marcos teóricos y metodológicos que permitan analizar herramientas varias del Internet, tales como los blogs, con diversos niveles de interacción e interactividad.
Una pregunta que queda sin respuesta, sin embargo, es si existe un fenómeno de dirección deliberada de la opinión en los espacios de discusión a través de blogs, y si éstos inciden directamente en la construcción de opinión pública. Para tal fin sería necesario dar un seguimiento más puntual al tipo de temáticas y argumentaciones que los medios periodísticos introducen en sus blogs. Ello permitiría determinar si las temáticas y la argumentación inducidas por los creadores de blogs favorecen la construcción de opinión en determinadas temáticas, si los medios tienden a perfeccionar y apropiarse de este recurso, o si los blogs se constituirán eventualmente como reductos de expresión pública auténticamente libre.

\section{REFERENCIAS BIBLIOGRÁFICAS}

Blood, R. (2000). Weblogs: a history and perspective. Rebbeca's pocket Weblog, 07/09/2000, consultado el 26 de marzo del 2009, y disponible en la siguiente dirección electrónica: http://www.rebeccablood.net/essays/ weblog_history.html

Branum, J. (2001). The Blogging Phenomenon: An Overview and Theoretical Consideration. San Marcos, TX: Southwest Texas University, consultado el 26 de marzo de 2009, y disponible en la siguiente dirección electrónica: http:// www.ajy.net/jmb/blogphenomenon.htm

Clark, J. (2001). Deconstructing "You've got blog". Fawny. Org. February, 24. 2001, consultado el 9 de junio de 2009, disponible en la siguiente dirección electrónica: http://fawny.org/decon-blog.html

Drezner, D. (2004). The power and politics of blogs. Trabajo presentado en la American Political Science Association, consultado el 26 de marzo de 2009, y disponible en la siguiente dirección electrónica: http://www. cs.duke.edu/courses/spring05/cps182s/readings/blogpowerpolitics.pdf

Dvorak, J. (2002). The blog phenomenon. PCMAG.com. The independent guide to technology en línea, consultado el 9 de junio de 2009, y disponible en la siguiente dirección electrónica: http://www.pcmag.com/article2/0,2817,12899,00.asp

Fumero, A. (2005). Un tutorial sobre blogs. El abecé del universo blog. TELOS, octubre-diciembre, 2005, consultado el 26 de marzo de 2009, disponible en la siguiente dirección electrónica: http://www.campusred.net/ telos/cuadernolimprimible.asp?idarticulo $=1$ Erev $=65$

Lasica, J. D. (2001). Blogging as a form of journalism. Online Journalism Review. May 24, 2001, USC Annenberg. Consultado el 9 de junio de 2009, disponible en la siguiente dirección electrónica: http://www.ojr.org/ojr/ workplace/1017958873.php

Orihuela, J. L. (2006). La Revolución de los Blogs. Cuando las bitácoras se convirtieron en el medio de comunicación de la gente. Madrid: La esfera de los libros. Libro electrónico consultado el 22 de junio de 2009, y disponible como eCuaderno en la siguiente dirección electrónica: http://www.ecuaderno.com/larevoluciondelosblogs/ 
Pérez Salazar, G. (2009). La construcción de Internet como medio de comunicación. Tesis de doctorado. México: Universidad Nacional Autónoma de México. Consultada el 22 de junio de 2009, y disponible en la siguiente dirección electrónica: http://www.inteligenciacolectiva.org/

Robinson, J. P. (1976). Interpersonal Influence in Election Campaigns: Two-sep flow Hypotheses. Public Opinion Quarterly, Vol.40, pp. 304-319.

Talbott, D. (2008). How Obama TReallyT Did ItThe social-networking strategy that took an obscure senator to the doors of the White House. MIT Technology Review September-October, 2008, consultado el 27 de marzo y disponible en la siguiente dirección electrónica: https://beta.technologyreview.com/web/21222/

Wacka, F. (2004). Beginners Guide to Corporate Blogging. Suecia, 2004, consultado el 10 de junio de 2009, disponible en la siguiente dirección electrónica: http://www.corporateblogging.info/basics/corporatebloggingprimer.pdf

Walker, R. (2005). La historia de los blogs 29/04/2005, consultado el 26 de marzo del 2009, y disponible en la siguiente dirección electrónica: http://www.rodrigowalker.cl/29-10-2005/la-historisi-de-los-blog/

Werbach, K. (2001). Triumph of the Weblogs. Steven Hatch`s blog. Consultado el 9 de junio de 2009, disponible en la siguiente dirección electrónica: http://www.hatch.org/2001/06/21/triumph-of-the-weblogs/

Wikipedia (2009). La Enciclopedia Libre. Weblogs and Jorn Barger. Consultada el 22 de junio de 2009, en la siguiente dirección electrónica: http://es.wikipedia.org/wiki/Jorn_Barger 